\title{
A case report on non surgical management of rarely found maxillary first molar with four roots
}

\author{
Babita Meena $^{1, *}$, Mukesh Hasija ${ }^{2}$ \\ ${ }^{1,2}$ Assistant Professor, Dept. of Conservative Dentistry and Endodontics, Faculty of Dentistry, Jamia Millia Islamia, New Delhi, \\ India
}

*Corresponding Author:

Email: bmeena@jmi.ac.in

\begin{abstract}
Endodontic mishaps are very unfortunate and most of the time iatrogenic Causes include inadequate knowledge of radicular morphology and some are due to the negligence of the dental surgeon. Maxillary first molar having three roots and three distinct canals is normal anatomical finding but presence of fourth root with a patent root canal is rare phenomenon to found in routine endodontic practice. Present case report discusses successful non surgical root canal treatment of maxillary first molar with a exceptional root canal anatomy of four separated roots. Invariably maxillary first molar has most complicated root canal anatomy. Most commonly found variation in maxillary molar are presence of two canals i.e.MB1 AND MB2 in mesiobuccal root and accessory canals in the area of furcation. Extra root or fourth root is a rare finding and least documented. This case report explains the successful root canal treatment of first maxillary molar with four distinct roots and four canals. Access cavity modified according to root canal anatomy, cleaning and shaping done with rotary instruments and obturation done with the help of calcium hydroxide based sealer.
\end{abstract}

Keywords: Four Roots, Endodontically, Irreversible Pulpitis, Heat Test, Periapical.

\section{Introduction}

Endodontic studies based on the complexities of root canal system always shown that a root with an ideal tapered canal and a centrally placed apical foramen is an exception which is supposed to be rule in endodontic practice. Based on investigative literature root canal morphology always has accessory canals and foramina in most of the teeth. Therefore a successful endodontist should have thorough anatomical knowledge of roots and root canals along with their anatomical variations. The success of root canal therapy depends upon an appropriate access cavity preparation and a good hermetic obturation. According to Ingle, for successful non surgical root canal treatment a good hermetic seal created by obturation is must which is not possible without proper cleaning and shaping of all radicular spaces. Lack of proper knowledge of the radicular anatomy can lead to several endodontic failures. Endodontic mishaps like missed roots and canals can lead to failure of endodontic therapies. A maxillary first molar presents vast range of anatomical variations in their radicular morphology. As demonstrated by Smadi and Khraisat the maxillary first molar has a high failure rate of orthograde non-surgical root canal treatment. The failure is mostly due to the presence of extra roots and canals that the clinician fails to negotiate, clean and seal. Fourth root or extra palatal root is rare variation from the usual three rooted morphology of maxillary first molar. Very few studies have been documented on uncommon four rooted morphology in maxillary first molars.

The following case report demonstrates diagnosis and successful non-surgical management of a rare case of maxillary first molar with two buccal and two distinct palatal roots.

\section{Case Report}

A 27 years old female patient with acute pain in right side of upper arch reported to clinic. Examination reveals acute pain in relation to maxillary first molar of right side due to deep occlusal caries and tooth was tender on percussion. Radiographic examination of 16 reveals radiolucency involving pulp and extra palatal root was clearly visible. Heat test gave positive response with lingering pain. No significant medical history was given by the patient. A symptomatic irreversible pulpitis was the diagnosis and treatment plan explained to the patient. Treatment was started after giving local anaesthesia and for ease of accessibility tooth was isolated with the help of rubber dam. For access cavity preparation the final outline form should be triangular in shape but in this case access opening done in rhomboidal shape to improve the visibility of extra canal orifice. Four separated root canal orifices seen on the pulpal floor. A dental operating microscope was used to detect and confirm the presence of fourth canal. It was presenting distal to the main palatal orifice and was located just below distal marginal ridge.

Working length was established using intra operative periapical radiographs (Fig. 1) and an apex locator (Root ZX, J. Morita Corp., Tokyo, Japan) was used to confirm the working length of root canals. Cleaning and shaping of all root canals was done using Protaper rotary instruments-6\% (Dentsply Maillefer, Switzerland) and canals were irrigated with $2.5 \%$ sodium hypochlorite with normal saline and $17 \%$ EDTA. Heat activation of irrigation solution was done. 
6\% Protaper gutta percha mastercones (Dentsply Maillefer, Switzerland) were selected (Fig. 2) and obturated (Fig. 3) with Sealapex sealer (SyberonEndo, Kerr, USA), sealer was placed in canals with help of lentulospirals. Restoration of the tooth was carried out using composite resin. Patient recalled after two month for review and tooth was asymptomatic.

\section{Discussion}

Anatomical variations in posterior teeth are enigma to endodontist. As radiographic image is only two dimensional it is very difficult to judge about exact number of root canals in posterior teeth. Although computerized digital radiographs can act as adjunct in such situations but for more precise diagnosis use of CBCT is mandatory. In the presented case all four root and root canals were clearly visible in diagnostic RVG therefore no further investigation were required. First maxillary molars commonly found as three rooted form as described by several anatomical studies. The fourrooted anatomy in maxillary first molar in its various forms is very rare and is more commonly found in the second or the third maxillary molar as described by various morphological studies.

Presented case discusses the presence of four separated roots as depicted by radiographic examination. According to literature four distinct roots in maxillary first molar is a rare to found phenomenon in routine endodontic practice. In order to negotiate extra canals, access cavity should be modified from usual outline form. Straight line access is must for good biomechanical preparation hence all undercuts should be removed. Irrigation of root canals is another important aspect of root canal treatment procedure because volume of tissue and debris will be more and comparatively inaccessible in such cases. Effervescent affect of $2.5 \%$ sodium hypochlorite solution will effectively remove organic content from all radicular spaces. Activation of irrigation is also helpful in removal of debris from all accessory canals.

Various studies done by Christie et al established that a maxillary molar with four roots is very rarely found phenomenon in routine endodontic practice. Yang et al CBCT studies also confirmed the rare occurrence of two separated palatal roots in maxillary molars. The study concluded that only as low as $1.12 \%$ cases have the prevalence of two palatal roots in maxillary second molar.

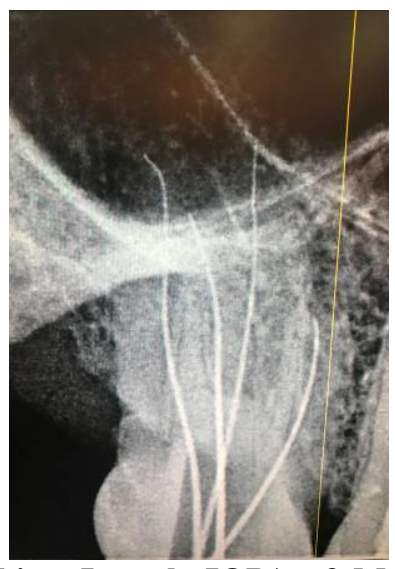

Fig. 1: Working Length IOPA of Maxillary First Molar (16)



Fig. 2: Master Cone IOPA of Maxillary First Molar (16)

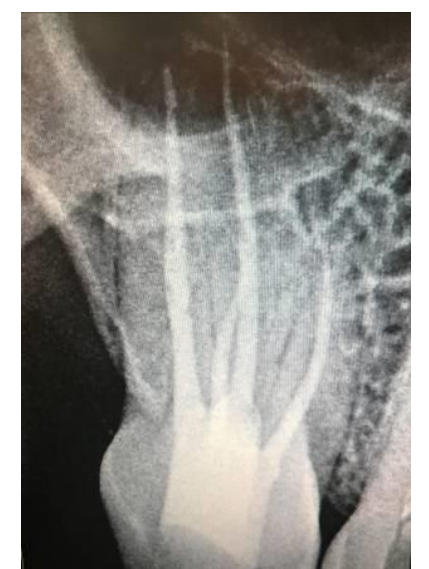

Fig. 3: Post Operative IOPA of Maxillary First Molar (16)

\section{Conclusions}

In the reported case pre- operative radiographs are important adjunct in finding extra root. Dentinal mapping at pulpal floor is another important guiding factor for locating extra or fourth canal orifice. Modification of access cavity preparation is required 
for such cases along with magnification, good illumination and intra operative radiographs with different angulations.

\section{References}

1. Smadi L, Khraisat A. Detection of a second mesiobuccal canal in the mesiobuccal roots of maxillary first molar teeth. Oral Surg Oral Med Oral Pathol Oral Radiol Endod 2007;103(3):e77-81.

2. F. Baratto-

Filho, L.F. Fariniuk, E.L. Ferreira, J.D. Pecora, A.M. Cru z-Filho, M.D. Sousa-Neto Clinical and macroscopic study of maxillary molars with two palatal roots, Int Endod $J$ 2002;35(9):796-801.

3. H. Jafarzadeh, M. Javidi, M. Zarei Endodontic retreatment of a maxillary second molar with three separate buccal roots Aust Endod J 2006;32(3):129-32.

4. S. J. Shin, J.W. Park, J.K. Lee, S.W. Hwang Unusual root canal anatomy in maxillary second molars: two case reports. Oral Surg Oral Med Oral Pathol Oral Radiol Endod 2007;104(6):e61-5

5. E. Deveaux Maxillary second molar with two palatal roots J Endod 1999;25(8):571-3.

6. S. Patel, P. Patel Endodontic management of maxillary second molar with two palatal roots: a report of two cases. Case Rep Dent 2012, 590406

7. M.B. Prashanth, P. Jain, P. PatniMaxillary right second molar with two palatal root canals, J Conserv Dent, 2010;13(2):94-6.

8. M.D. Peikoff, W.H. Christie, H.M. Fogel, The maxillary second molar: variations in the number of roots and canals, Int Endod J 1996;29(6):365-9.

9. W.H. Christie, M.D. Peikoff, H.M. Fogel, Maxillary molars with two palatal roots: a retrospective clinical study. J Endod 1991;17(2):80-4.

10. B. Yang, Q. Lu, Q.X. Bai, Y. Zhang, X.J. Liu, Z.J. LiuEva luation of the prevalence of the maxillary molars with two palatal roots by cone-beam CT, Zhonghua Kou Qiang Yi Xue Za Zhi 2013;48(6):359-62.

11. R. Jaya, R.S. Mohan Kumar, R. SrinivasanA rare case of dilated invaginated odontome with talon cusp in a permanent maxillary central incisor diagnosed by cone beam computed tomography, Imaging $S c i$ Dent 2013;43(3):209-13. 